\title{
S DOR-TYPE VARIABLES IN OTHER GALAXIES
}

\author{
A. S. SHAROV \\ Sternberg Astronomical Institute, Moscow University, Moscow, U.S.S.R.
}

\begin{abstract}
The variability of these objects, almost the brightest ones found in galaxies, is discussed, followed by a description of colour indices and spectra. In the last sections the classification problems and items for further investigation are mentioned.
\end{abstract}

The brightest objects in galaxies, along with supernovae and novae at maximum luminosity and globular clusters, are irregular variables which are often called HubbleSandage stars. Their investigation is of great interest in several respects. Luminous stars have short lives, and here we probably have objects at a rapid evolutionary stage. Papers by Ledoux (1941) and Schwarzschild and Härm (1959) show that massive stars cannot be stable objects. It is supposed that the variables under discussion may, in certain respects, correspond to these theoretical considerations. Finally, high luminosity makes these stars possible indicators of distances of galaxies.

\section{Variability of Brightness}

The first variables of this type were discovered more than fifty years ago independently by Duncan (1922) and Wolf (1923) in the Triangulum galaxy M33. Now they are also known in the Andromeda galaxy M31 and the galaxy NGC 2403. The discovery of similar objects was also reported for the galaxies M81 (Tammann and Sandage, 1968) and M 101 and NGC 4486 (Hubble and Sandage, 1953); however, no details or lists of objects were published.

The variables already studied are listed in Table I, where the names of discoverers and the authors of photometric investigations are also given. Question marks indicate stars which are only possible members of the type. The similarity of AE And to other bright variables in M 31 was supposed by Luyten and recently confirmed by the author.

The luminosity of bright irregular variables usually varies by from one to several magnitudes, and it reaches at maximum light $M_{B}=-11^{m} 3$ to $-7^{m} .1$. From this point, the stars V 14, 52 and 53 in NGC 2403 may belong to another type since their light variations do not exceed $0^{m} 5$.

In Hubble's opinion (1929), one possibly should attribute to this type several stars given in his list of M 31 novae (Nos. 26, 36 and 39). They were visible for several years, but they did not reach the luminosity of other bright irregular variables in $\mathbf{M} 31$. These stars may also be slow novae.

Schematic light curves of variables in M 31, M 33 and NGC 2403 are shown in Figure 1 (the 3 small-amplitude objects in NGC 2403 are omitted).

For the stars in M 31 and M 33 the light curves are plotted on the basis of data by Hubble (1929), Hubble and Sandage (1953), Meinunger (1971a), Rosino and Bianchini (1973) and the author (Sharov, 1973); for the stars in NGC 2403 the light curves are 
TABLE I

List of bright irregular variables in M 31, M 33 and NGC 2403

\begin{tabular}{|c|c|c|}
\hline \multicolumn{2}{|c|}{ Var } & \multirow{2}{*}{$\begin{array}{l}\text { Investigators } \\
\text { Hubble (1929), Rosino and Bianchini (1973), Sharov (1973) }\end{array}$} \\
\hline M 31 & V 15 & \\
\hline & $\mathrm{V} 19=\mathrm{AF}$ And & $\begin{array}{l}\text { Luyten (1927), Hubble (1929), Hubble and Sandage (1953), Meinun- } \\
\text { ger (1971a), Rosino and Bianchini (1973), Sharov (1973) }\end{array}$ \\
\hline & AE And & Luyten (1928), Meinunger (1971a), Sharov (1973) \\
\hline & Var A-1 & Rosino and Bianchini (1973), Sharov (1973) \\
\hline \multirow[t]{5}{*}{ M 33} & Var 1 & Duncan (1922), Hubble (1926), Rosino and Bianchini (1973) \\
\hline & Var 2 & $\begin{array}{l}\text { Duncan (1922), Wolf (1923), Hubble (1926), Hubble and Sandage } \\
\text { (1953), Rosino and Bianchini (1973), Sharov (1973) }\end{array}$ \\
\hline & Var A & $\begin{array}{l}\text { Hubble and Sandage (1953), Rosino and Bianchini (1973), Sharov } \\
\text { (1973) }\end{array}$ \\
\hline & Var B & $\begin{array}{l}\text { Hubble and Sandage (1953), Rosino and Bianchini (1973), Sharov } \\
\text { (1973) }\end{array}$ \\
\hline & Var C & $\begin{array}{l}\text { Hubble and Sandage (1953), Rosino and Bianchini (1973), Sharov } \\
\text { (1973) }\end{array}$ \\
\hline \multirow[t]{8}{*}{ NGC 2403} & V 12 & Tammann and Sandage (1968) \\
\hline & V $14(?)$ & Tammann and Sandage (1968) \\
\hline & V 22 & Tammann and Sandage (1968) \\
\hline & V 35 & Tammann and Sandage (1968) \\
\hline & V 37 & Tammann and Sandage (1968) \\
\hline & V 38 & Tammann and Sandage (1968) \\
\hline & V $52(?)$ & Tammann and Sandage (1968) \\
\hline & V $53(?)$ & Tammann and Sandage (1968) \\
\hline
\end{tabular}

plotted using data by Tammann and Sandage (1968). Solid lines show well-observed fractions of light curves, and dashed lines show the possible appearance of light curves for those intervals when the stars were not observed sufficiently. Individual brightness estimates are also plotted if they are significant for tracing the light curve; among these are some estimates from old photographic atlases. The curves are illustrative only, and the photometric scales were not transformed into a homogeneous systems. In some cases this is impossible to do since the magnitudes of comparison stars were not published. There even exists a difference between the light curves of AF And during 1910-1920 in the papers by Hubble (1929) and Hubble and Sandage (1953). The magnitudes of comparison stars for this variable seem to need significant corrections. In Figure 2 we compare the photographic magnitudes of four comparison stars (Nos. 9, 14, 16, and 19) according to Hubble and Sandage (1953) and the magnitudes found by the author. The latter are in good accordance with Arp's (1956) photoelectric values.

We have attempted to separate all the light curves into groups on the grounds of the character of variability. This was done for better perception only and cannot be a basis for detailed classification; for some stars the observations are rather fragmentary which also makes classification impossible.

There is a wide variety of light curves. Among the variables there exist objects with a single large brightness oscillation as well as objects with numerous oscillations; some stars were constant in light for a long time. In spite of the long period of observations $(50-70 \mathrm{yr})$, the character of variability is not clear in all details. Particularly, it is 


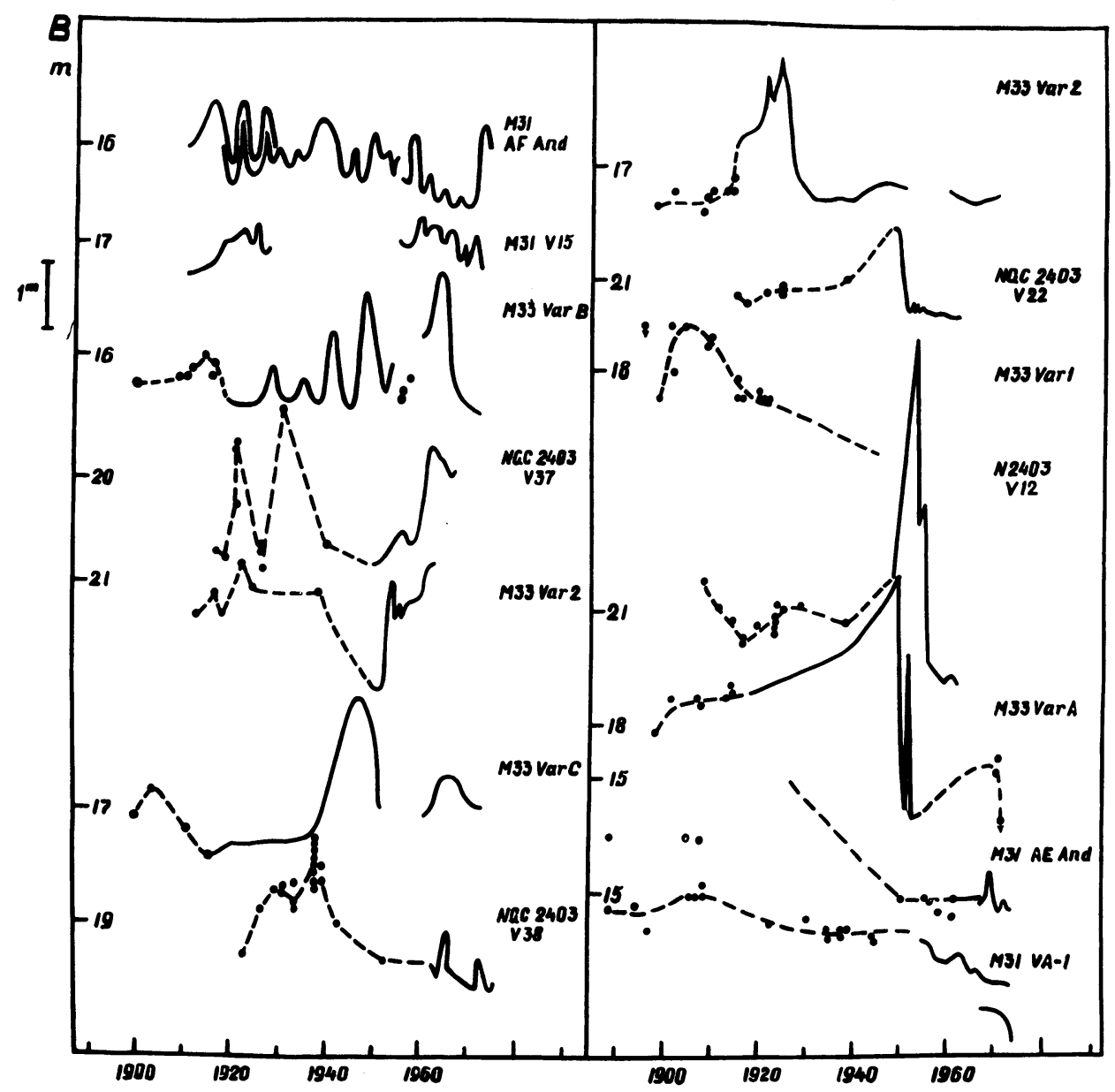

Fig. 1. Light curves of bright irregular variables in M 31, M 33 and NGC 2403.

not known whether the absolute maxima and minima are covered by observations or not, so from this point the question of utilization of the variables as reliable distance indicators is still open.

\section{Colour Indices}

In order to study the nature of the variables under consideration, it is very important to know their colours which give some general information on the energy distribution in their spectra.

Up to now colour indices of these variables (CI or $B-V$ ) were determined only on the basis of magnitude estimates made by eye on plates taken in blue and yellow light (Hubble and Sandage, 1953; Tammann and Sandage, 1968; Rosino and Bianchini, 1973). There were no determinations of $U-B$. In the autumn of 1973 (J. D. 2441928 ), photoelectric measurements were carried out for the first time for 3 variables in M 31 

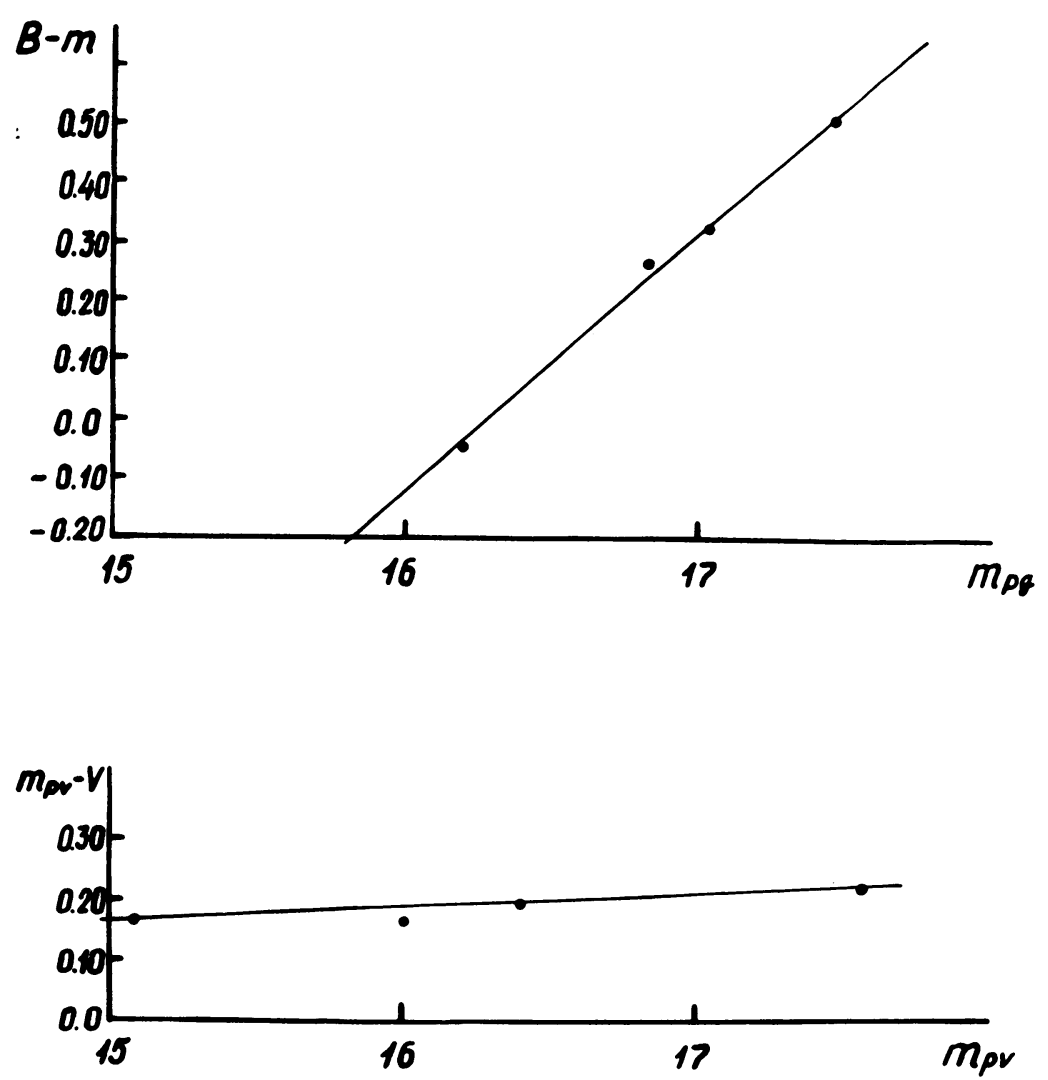

Fig. 2. Comparison of photographic magnitudes of comparison stars near AF And (Var 19) obtained by Hubble and Sandage and by the author.

with the $2.6 \mathrm{~m}$ Shajn telescope of the Crimean Astrophysical Observatory (Sharov et al., 1975). The results are given in Table II. (The author's photographic measurements in the UBV system (Sharov, 1973) should be considered only as preliminary ones.) Positions of the stars on the two-colour diagram $(U-B)$ versus $(B-V)$ are shown in Figure 3 (circles). Several galactic variables which are discussed later are also shown. The main sequence and the sequence of supergiants are shown by thick and thin lines, respectively. The arrow heads show the positions of the variables corrected for interstellar absorption in the Galaxy in the direction of M 31 (McClure and Racine, 1969). On the two-colour diagram the variables are situated in the area of

\section{TABLE II}

Photoelectric UBV photometry of bright irregular variables in M 31

\begin{tabular}{lllll}
\hline Var & $V$ & $B-V$ & $U-B$ & $Q$ \\
\hline AE And & $177^{m} 03$ & $+0 m 06$ & -0.76 & -0.98 \\
AF And & 16.63 & +0.14 & -0.99 & -1.09 \\
Var A-1 & 16.90 & +0.31 & -0.64 & -0.86 \\
\hline
\end{tabular}


somewhat reddened early type stars; the reddening is probably caused by absorbing matter in M 31. The colours of AE And which is situated outside the spiral arms of M 31 are near the normal colours of early type supergiants. Faint hydrogen emissions that are observed in the spectra of the variables cannot change their colours in the UBV system considerably.



Fig. 3. Two-colour diagram $(U-B)$ versus $(B-V)$ for bright irregular variables in $\mathrm{M} 31$, $\mathrm{S}$ Dor and some galactic variables.

Let us consider the available data on the colours of the variables. According to the above-mentioned papers, the colour indices of the variables in $M 31$ and $M 33$ are near zero and even negative. Var A in M 33 presents an exception; its colour index. changed from +0.3 to about $+1^{m} \cdot 5$ during two years of rapid decrease of brightness. Up to now no explanation of this phenomenon has been proposed. It is not excluded that the symbiotic nature of the variable might be the reason; then at different brightnesses, either the white or red component plays the main rôle. However, first of all it is necessary to be quite assured of the correctness of the comparison stars scale for the variable. It would be very desirable to establish a photoelectric sequence near the variable and to carry out new brightness estimates on Hubble and Sandage's plates.

It seems to us that the determinations of colours of variable stars in NGC 2403 are not reliable. According to Tammann and Sandage (1968), the bluest $(B-V)$ values for the stars V 37 and V 38 are -0.42 and -0 . 72 . Such colours are met only among novae and are conditioned mainly by strong emissions and not by continuous spec- 
trum. By analogy with the stars in M 31 and M 33 there is no reason to expect any very strong emissions for the stars in NGC 2403. Considerable colour variations are also doubtful. For instance, the authors give for V 12 and $\mathrm{V} 35$ at different times the following $(B-V)$ values: $+0 .^{m} 04$ to $+1^{m} 15$ and -0.26 to $+0^{m} .80$. If we select from the published material only the values obtained from pairs of plates taken during one night, and moreover, take only the most accurate values of these, then we find (with a small number of exceptions) some synchronism in colour variations which indicates systematic errors in the photometry (Figure 4).

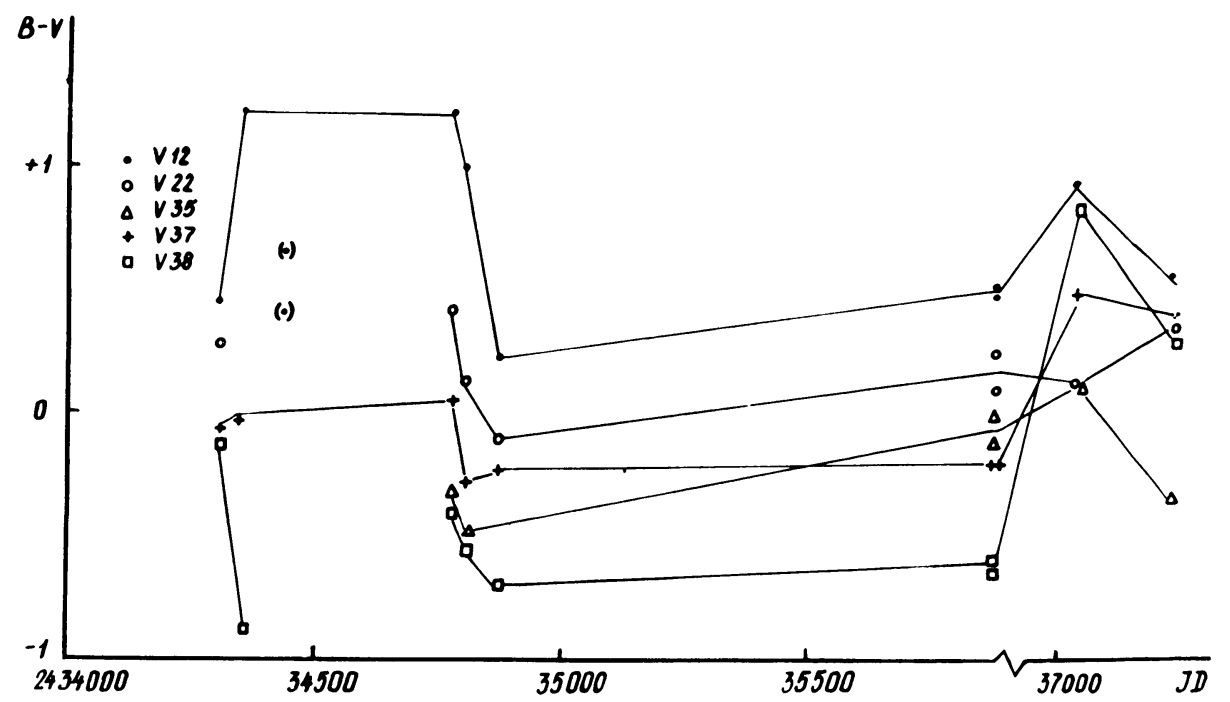

Fig. 4. Synchronism in color variations of some variables in NGC 2403.

Further, we draw an analogy between these variables and the stars S Dor, AG Car and HR Car. Their variations of brightness are not accompanied by such significant colour changes. For instance, in 1953-1955 the colour index of S Dor in the Cape system remained constant during a $0^{m} .8$ decrease in light (Wesselink, 1956). Later Feast et al., (1960) noticed a change of $(B-V)$ colour from +0.22 during maximum light to $+0{ }^{m} 10$ during minimum light. Colour variations of AG Car and HR Car are very small (Wisse and Wisse, 1971).

\section{Spectra of Bright Irregular Variables}

Up to now the data on spectra were published for only four variables: AF And (V 19) in M 31 and Var A, Var B and Var C in M 33; the spectra were photographed at Mt Wilson observatory in 1940-1952 (Hubble and Sandage, 1953). The spectrograms were obtained with a dispersion of $220-430 \AA \mathrm{mm}^{-1}$ at $\mathrm{H} \gamma$ which is insufficient for detailed analysis. So the spectral information on this interesting type of variable stars is rather scanty. 
Hydrogen lines of variable intensity, usually in emission and sometimes in absorption, are present in the spectra. P Cyg profiles were noticed for hydrogen lines in the spectra of Var A and Var B in M 33. For Var B neutral helium emission lines He I 3889 and HeI $4471 \AA$ were also found. These data may be interpreted as evidence for the early spectral type of the stars. On the other hand, the investigators also mentioned $\mathrm{H}$ and $\mathrm{K} \mathrm{Ca}$ II absorption lines of variable intensity. The Mg I triplet 3890, 3892 and $3896 \AA$ and the FeII lines 4171 and $4177 \AA$ which are the criterion of high luminosity were found on the best spectrogram of Var $\mathrm{A}$. On the grounds of the $\mathrm{H}$ and $\mathrm{K} \mathrm{Ca} \mathrm{II}$ lines and the intense continuum in the blue and ultraviolet region, Hubble and Sandage attributed these variables to intermediate $F$ type.

In the autumn of 1973 we obtained the spectra of AE And, AF And and Var A-1 in $M 31$ and Var C in M 33 in the region $\lambda$ 5500-7500 $\AA$. The observations were made using the grating spectrograph with a contact image tube installed at the Cassegrain focus of the $125 \mathrm{~cm}$ reflector of the Sternberg Institute Crimean station. The dispersion was about $230 \AA \mathrm{mm}^{-1}$; the spectral resolution was $10 \AA$. Strong $\mathrm{H} \alpha$ emission lines were found for all the stars. The radial velocities confirmed that the stars are members of the corresponding galaxies.

\section{Classification of Bright Irregular Variables}

Hubble and Sandage were the first to express the idea that irregular variables in M 31 and $\mathbf{M} 33$ and S Dor in the LMC, which are P Cyg type stars according to spectral features, belong to a single type. The only objection against it was that, according to Gaposchkin (1942), S Dor might be an eclipsing binary. But further observations did not confirm that result (Smith, 1954a; Wesselink, 1956; Alexander and Thackeray, 1971). Some considerations on the similarity of $S$ Dor to bright variables in other galaxies were also given by Smith (1954b), Wesselink (1956) and Gratton (1963). It seems to us that these considerations have serious grounds.

The problem of classification for these variable stars may be solved on the basis of the following main features: (1) light curves, (2) luminosities, and (3) colour indices and spectra.

Figure 5 shows the light curves of two galactic stars, HR Car and AG Car, and also of S Dor; these stars, together with other objects, according to Meinunger (1971b) form the P Cyg type of variable stars. (In order to distinguish the phenomenon of variability from spectral features the authors of the General Catalogue of Variable Stars gave this type another name, $\mathrm{S}$ Dor.) The light curves were plotted according to the following sources of data: AG Car - Greenstein (1938), Mayall (1969); HR Car Hoffleit (1940); S Dor - Alexander and Thackeray (1971), Appenzeller (1972). There was no reduction to a single scale, and for AG Car the curve consists of two parts based on photographic and visual observations. The general similarity of the curves in Figures 1 and 5 is obvious.

We have already discussed the absolute magnitudes of bright variables in external galaxies. The absolute magnitudes of AG Car, HR Car and P Cyg are the same 
$\left(M_{V}=-8^{m} \cdot 0\right.$ to $\left.-8^{m} \cdot 5\right)($ Viotti, 1971). The star P Cyg during maximum light was comparable to the brightest variables in external galaxies.

Now we return to the two-colour diagram, where, apart from M 31 stars, S Dor and analogous variables of Galaxy were also plotted. For $\mathbf{S}$ Dor mean colour values are according to Alexander and Thackeray (1971), and for the rest of the stars according to the compilation by Viotti (1971). The diagram shows that all the stars under consideration occupy the same region in the two-colour diagram and hence have similar energy distributions in their continuous spectra.



Fig. 5. Light curves of AG Car, HR Car and S Dor

The spectra of variables in M 31 and M 33 may not be regarded as sufficiently well studied for detailed comparison with the spectra of galactic stars and $\mathbf{S}$ Dor. However, it is necessary to emphasize that all these stars have hydrogen emission lines with $P$ Cyg profiles. Note that it is the investigation of the spectrum and brightness variations of S Dor that enabled Wesselink (1956) to point out this star's similarity to the variables in $M 31$ and $M 33$.

Recently Thackeray (1974) again stated the similarity of $S$ Dor to the variables in $M 31$ and M 33 with respect to luminosity and the character of brightness variations. But, in his opinion, the variables in $M 31$ and $M 33$ have a wider range of colours, they are redder and become still redder during maximum light, and their spectra belong to later types. The photoelectric observations presented above show the absolute accordance of the colours of S Dor with those of the stars investigated in M 31 
and $M$ 33. We do not yet have reliable information on the relation of colour variations to the brightness variations. We have already stated that detailed spectroscopic investigation is necessary.

\section{Some Problems for Further Investigation}

One should recognize that high luminosity variables in external galaxies are still insufficiently investigated. The character of their variability requires prolonged series of photometric, colorimetric and spectroscopic observations. In particular, it would be very important to establish the absolute light maxima, having in mind the possible use of these stars as distance indicators. Of course, the observations should be in modern photometric systems; photoelectric sequences should be established near each star for this purpose. This would also make it possible to revise the observational material obtained earlier.

The determination of colour indices and the search for their possible variations also present an interesting problem. Such observations should be done only by photoelectric technique.

It is necessary to use the largest telescopes for detailed spectroscopic investigations. It is very important for photometric and spectroscopic observations to be simultaneous.

One should also pay attention to the necessity of the search for new luminous irregular variables in the Galaxy and in external galaxies.

\section{References}

Alexander, J. B. and Thackeray, A. D.: 1971, Observatory 91, 25.

Appenzeller, I.: 1972, Publ. Astron. Soc. Japan 24, 483.

Arp, H. C.: 1956, Astron. J. 65, 15.

Duncan, J. C.: 1922, Publ. Astron. Soc. Pacific 34, 290.

Feast, M. W., Thackeray, A. D., and Wesselink, A. J.: 1960, Monthly Notices Roy. Astron. Soc. 121, 354.

Gaposchkin, S.: 1942, Publ. Astron. Soc. Pacific 54, 264.

Gratton, L. 1963, 'The Problem of Eta Carinae,' in Proc. Int. School of Physics 'Enrico Fermi', Course

XXVIII (ed. by L. Gratton, Academic Press, New York-London, p. 297.

Greenstein, N. K.: 1938, Harvard Obs. Bull. 908, 25.

Hoffleit, D.: 1940, Harvard Obs. Bull. 913, 4.

Hubble, E. P.: 1926, Astrophys. J. 63, 236.

Hubble, E. P.: 1929, Astrophys. J. 69, 103.

Hubble, E. P. and Sandage, A. R.: 1953, Astrophys. J. 118, 353.

Ledoux, P.: 1941, Astrophys. J. 94, 537.

Luyten, W. J.: 1927, Harvard Obs. Bull. 851, 4.

Luyten, W. J.: 1928, Harvard Obs. Bull. 859, 1.

Mayall, M. W.: 1969, Observatory 63, 221.

McClure, R. D. and Racine, R.: 1969, Astron. J. 74, 1000.

Meinunger, L.: 1971a, Mitt. Veränd. Sterne Sonneberg 5, 177.

Meinunger, L.: 1971b, Veröff. Sonneberg 8, No. 1, 1.

Rosino, L. and Bianchini, A.: 1973, Astron. Astrophys. 22, 453.

Schwarzschild, M. and Härm, R.: 1959, Astrophys. J. 129, 637.

Sharov, A. S.: 1973, Perem. Zvezdy 19, 3.

Sharov, A. S., Lyuty, V. M., and Esipov, V. F.: 1975, Pis'ma Letters Astron. Zh. (in press). 
Smith, H. J.: 1954a, Australian J. Sci. Suppl. 17, 18.

Smith, H. J.: 1954b, Astron. J. 59, 332.

Tammann, G. A. and Sandage, A. R.: 1968, Astrophys. J. 151, 825.

Thackeray, A. D.: 1974, Monthly Notices Roy. Astron. Soc. 168, 221.

Viotti, R.: 1971, Publ. Astron. Soc. Pacific 83, 171.

Wesselink, A. J.: 1956, Monthly Notices Roy. Astron. Soc. 116, 3.

Wisse, P. N. J. and Wisse, M.: 1971, Astron. Astrophys. 12, 149.

Wolf, M.: 1923, Astron. Nachr. 217, 475. 